\title{
¿DOS VERBOS LLEGAR EN ESPAÑOL?
}

TWO VERBS LLEGAR IN SPANISH?

\author{
Fernando López García \\ Universidad Complutense de Madrid \\ ferkiinmadrid@hotmail.es
}

Recibido: 07/10/2015

Aceptado: 16/05/2016

\begin{abstract}
Resumen
Nuestro objetivo es demostrar que en español el verbo llegar posee dos interpretaciones en función del Aspecto de los predicados en los que aparece: inacusativa combinado con sujetos no volitivos e inergativa combinado con sujetos volitivos. Primero esbozaremos el fenómeno de la Inacusatividad y describiremos los grupos VCE y VEA. A continuación, propondremos una justificada reducción del grupo de los VM a cuatro verbos semántico-cognitivamente primitivos, entre los que se encuentra llegar, para finalmente exponer las pruebas que sustentan sus dos posibles interpretaciones.

PALABRAS CLAVE: inacusatividad, llegar, verbos de existencia o aparición, verbos de movimiento, aspecto léxico.
\end{abstract}

\begin{abstract}
Our aim is to prove that the verb 'llegar' (arrive) possesses two possible interpretations in Spanish according to the lexical aspect of the predicates in which appears: unaccusative, combined with no-volitional subjects and unergative, combined with volitional subjects. Firstly, we will outline the Unaccusativity phenomenon and we will describe the VCS (Verbs of Change of State) and VEA (Verbs of Existence or Appearance) groups. Later on, we will propose a justified reduction of MV (Motion Verbs) group to four primitive semanticcognitively verbs, among which is the verb 'llegar', in order to present, finally, the evidences that underpin its two possible interpretations.

KEYWORDS: unaccusativity, llegar, verbs of existence or appearance, motion verbs, lexical aspect.
\end{abstract}

\section{Introducción*}

Las particulares características que presenta el verbo llegar en español lo convierten en un elemento ciertamente complejo para su análisis dentro de un grupo delimitado. Así, entraña cierta dificultad aseverar con rotundidad que dicho verbo pertenezca al grupo de los

\footnotetext{
* Queremos agradecer a María Jesús Fernández Leborans, Luis García Fernández, Cristina Sánchez López y Luis Sáez del Álamo, así como a dos revisores anónimos, sus observaciones y sus consejos durante la elaboración de una versión previa de este trabajo.
}

Para citar este artículo / To cite this article: López García, Fernando (2016). ¿Dos verbos llegar en español? ELUA, 30: 163-180. doi:10.14198/ELUA2016.30.08

Enlace / Link: http://dx.doi.org/10.14198/ELUA2016.30.08 
llamados Verbos de Cambio de Estado (VCE) o al grupo de los llamados Verbos de Existencia o Aparición (VEA). De igual forma, es difícil asumir que el verbo llegar se comporta como los demás Verbos de Movimiento (VM). Entonces, lo primero que debemos hacer es intentar explicar por qué este verbo se separa de todos aquellos grupos en los que se le tiende a encuadrar, tarea para la que previamente debemos detenernos en describir tales grupos.

\subsection{Unas notas sobre Inacusatividad}

La intransitividad verbal ha sido uno de los campos de estudio más productivos para la investigación gramatical y semántica en las últimas tres décadas, debido a la amplia variedad de predicados que abarca. La principal distinción entre tales predicados, comúnmente aceptada, establece dos clases de verbos intransitivos: ergativos o inacusativos e inergativos o intransitivos puros. La diferencia entre unos y otros se pone de manifiesto en los precursores trabajos de Perlmutter (1978) y de Burzio (1981, 1986), que postulan la llamada Hipótesis Inacusativa a partir de la caracterización de la sintaxis de las oraciones pasivas impersonales. Así, Perlmutter (1978: 160) intuye que «certain intransitive clauses have an initial 2 but no initial 1», entendiendo por 2 argumento interno y por 1, argumento interno:

(1)

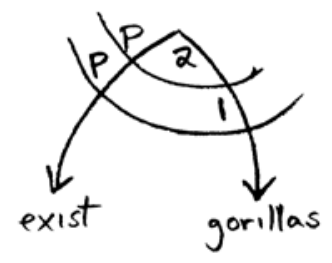

Al margen de la representación formal de Perlmutter, que tendría su equivalente generativista ${ }^{1}$ en (2), lo que precisa el autor es que hay ciertos verbos que seleccionan argumento interno, pero no externo:

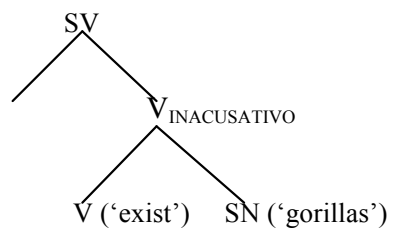

Los verbos que presentan una configuración como la reproducida en (1) y (2) son verbos ergativos o inacusativos, característica que se sustenta en la denominada Generalización de Burzio (tomado de Bosque y Gutiérrez-Rexach, 2009: 397):

a. Un verbo asigna caso a la posición de objeto (o complemento directo) solo si asigna papel temático a la posición de sujeto.

b. $[-\theta] \mathrm{V}[-\mathrm{C}]$

1 El estudio de Perlmutter se encuadra en la Gramática Relacional, mientras que el de Burzio pertenece al marco de la Gramática Generativa (Modelo de la Rección y el Ligamiento o de Principios y Parámetros). 
Como consecuencia de ello, se deduce una asimetría en las etiquetas que uno y otro tipo de verbos emplean en la caracterización semántica de sus argumentos: los inacusativos seleccionan un actante Tema o Paciente y los inergativos, uno Agente. En palabras de Torrego (1989: 253): «The single argument of unaccusative verbs, unlike that of unergative verbs, is base-generated in the structural object position at D-structure. The thematic role of unaccusative verbs is generally theme or patient, while the argument of unergative tends to be agentive». Se ha señalado en varias ocasiones la cercanía de los verbos inergativos a los verbos transitivos en algunas de sus propiedades. La más interesante para nuestro estudio es la que pone en conexión su único argumento descrito como sujeto Agente y el argumento externo de los transitivos, habitualmente también agentivo ${ }^{2}$.

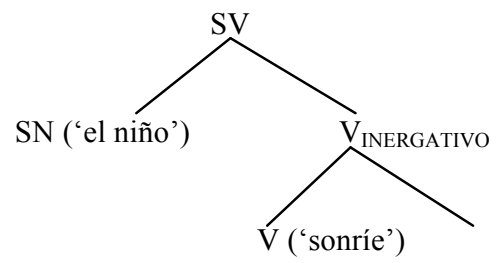

La base de la inacusatividad se inscribe, pues, en la no manifestación de Agente en la estructura léxico-sintáctica del núcleo verbal, relacionada con el hecho de que la sintaxis de las construcciones pasivas es inacusativa.

Villar (1983: 12) cree oportuno sustituir los términos transitivo e intransitivo por activo e inactivo, pues algunas lenguas no contemplan la noción de transitividad en su gramática. Si bien en español estos términos pueden solaparse con los empleados en otros ámbitos de la lingüística, valoramos positivamente su uso para la explicación de los comportamientos inergativo e inacusativo respectivamente. Cabe señalar que hay verbos activos monovalentes (Alberto nada) y verbos inactivos bivalentes (Alberto sufre migrañas), por lo que los conceptos de transitividad y actividad no son intercambiables desde el punto de vista sintáctico, pero lo son desde su perspectiva semántica, que es la que nos interesa para interpretar la inacusatividad en su caracterización primaria, pues la actividad se corresponde con la implicación de un sujeto Agente en el evento. Yendo un paso más allá, conviene tener en cuenta que la inacusatividad es, como dice Villar (1983: 45), «un fenómeno gradual», hecho que se aprecia muy bien en el español, que cuenta con verbos puramente inacusativos (crecer), verbos puramente inergativos (sonreír) y verbos que pueden presentar una u otra cualidad a partir de ciertos mecanismos sintácticos que ejercen coacción sobre su léxico (salir). A diferencia de otras lenguas, como el vasco, dotada de morfología ergativa, o el francés, básicamente nominativo-acusativa, el español se sirve de la sintaxis para reflejar el paso de ergatividad a inergatividad. De esto último se deduce que la ergatividad es primitiva y la inergatividad, derivada en nuestra lengua.

A continuación vamos a exponer algunas de las pruebas que conforman un diagnóstico de Inacusatividad para el español, basado en los trabajos de Torrego (1989), Cifuentes Honrubia (1999), Mendikoetxea (1999), Batiukova (2004), Fernández Leborans (2005), Pérez Jiménez

2 Chomsky (2006 y 2013) vincula construcciones transitivas e inergativas con la proyección funcional $v^{*}$ y construcciones inacusativas y pasivas con la proyección funcional $v$. 
y Moreno Quibén (2007), Bosque y Gutiérrez-Rexach (2009) y Cano (2010), lo que nos permitirá establecer con mayor precisión la distinción entre predicados inacusativos e inergativos:

i) Formación de cláusulas de participio absoluto, construcciones que expresan la situación de un evento, el correspondiente a la cláusula de participio absoluto, en un momento temporalmente anterior al evento principal. Dicha cláusula se forma en torno a la concordancia del argumento (externo, el sujeto natural en el caso de los inergativos, o interno, el sujeto derivado de los inacusativos) con el participio del verbo que introduce el primer evento; el contraste se produce entre la aceptabilidad de la construcción con predicados inacusativos -véase (4) - y su agramaticalidad con predicados inergativos -véase (5)-:

(4) a. Crecido el árbol, el ayuntamiento acordonó la zona

b. Caído el muro, la fortaleza se volvió vulnerable

c. Muerto el perro, se acabó la rabia

(5) a. *Sonreído Juan, mejoró el rendimiento colectivo

b. *Bailada Cristina, la fiesta continuó hasta el amanecer

c. *Luchado el ejército, la retirada fue inmediata

Nótese que la restricción que impone esta prueba respecto a los predicados inergativos -sumada a la diferencia interno-externo en la semántica del Objeto del predicado- tiene que ver con su atelicidad, esto es, con su no-delimitación. Los predicados inergativos, en tanto que [+ durativos] / [- télicos], no expresan una culminación, por lo que es inviable la existencia del segundo evento al no resultar concluido el primero, el expresado por la cláusula.

ii) Formación de cláusulas no flexivas que modifican a un SN: los predicados inacusativos pueden expresar estas construcciones -véase (6)-, no así los inergativos -véase (7)-:

(6) a. El árbol crecido

b. La muralla caída

c. El perro muerto

(7) a. *El chico sonreido

b. *La mujer bailada

c. *El ejército luchado

Asimismo, obsérvese la aceptabilidad de participios femeninos con verbos inacusativos, opción no probable en el caso de los inergativos: la muralla caída, la carta llegada, la niña recién nacida, etc. vs. *la mujer bailada, *la perla brillada, *la niña sonreída, etc.

iii) Construcciones con sujeto escueto o sin determinación con verbos inacusativos. Estos sujetos son SSNN que carecen de determinante que los introduzca y adoptan una posición obligatoriamente postverbal -véase (8), cf. con (9)-:

(8) a. Nacen niños

b. Florecen cerezos

c. Arde paja 
(9) a. *Corren jugadores

b. *Trabajan obreros

c. *Sonrien alumnos

Algunos autores consideran que esta prueba no resulta válida si se introducen complementos adjuntos de índole espacio-temporal en posición preverbal en los casos de sujeto inergativo, (v. Torrego, 1989: 258-259), puesto que neutralizan su sentido agentivo: $\underline{A}$ lo lejos corren jugadores, En esta ciudad trabajan obreros. Otros autores, sin embargo, creen que este tipo de secuencias constituyen un contraargumento falso a la prueba presentada; este punto de vista se sustenta principalmente en la reinterpretación que parece sufrir el predicado inergativo, que se acerca más a uno de tipo existencial o de percepción (inacusativos) que a uno agentivo, teniendo en cuenta la ya de por sí dudosa gramaticalidad de ciertas oraciones: A lo lejos corren jugadores $\rightarrow$ "Se ven jugadores a lo lejos"; En esta ciudad trabajan obreros $\rightarrow$ "Hay obreros en esta ciudad".

En nuestra opinión, la prueba es sólida y no se cancela al añadir un contexto en el que se desarrolla el evento expresado por los respectivos predicados, pues la relación que se establece entre el sujeto escueto y el predicado se da a través de posiciones estructurales diferentes según el argumento sea interno o externo, haya o no complementos con los que se pretenda reinterpretarlos: la posición del argumento de los inacusativos es, de forma canónica, la de Objeto profundo, mientras que la del argumento externo de los inergativos es derivada sintácticamente en este caso y no puede tomarse como idéntica a la de Objeto profundo, sino que se trata de un Objeto superficial, generado en la sintaxis, que requiere de determinación para ocupar una posición postverbal; por su parte, el argumento interno de los inacusativos no necesita estar determinado para permanecer en su posición natural:
a. Arg. Ext. - Arg. Inter. derivado - SV - Arg. Ext. derivado - Arg. Inter.
b. Los jugadores
corren
c.
Corren los jugadores
d.
*Corren jugadores
e.
$\underline{\text { Los niños }}$
nacen
f.
Nacen
los niños
g.
Nacen
niños

A esta razón hay que añadirle otra de carácter aspectual: la agentividad queda anulada en pos de la telicidad que denota el conjunto formado por la posición interna y la ausencia de determinación; así, en Corren jugadores, por ejemplo, no resulta informativa la interpretación de Actividad, dado que se trata de una interpretación puntual, relacionada con una lectura de evento de acaecimiento (inacusativo).

iv) Incorporación del adverbio átono ahí usado de forma expletiva o no referencial en secuencias inacusativas -véase (10)-:

(10) Ahí nacen niños vs. *Ahí corren niños 
Véase Deal (2009) para un análisis del uso del adverbio inglés there relacionado con esta prueba.

v) Creación de adjetivos deverbales acabados en -ble por parte de los inacusativos -véase (11)- vs. formación de sustantivos de Actividad con el sufijo -dor y de participios de presente con el sufijo -nte, solo viables con verbos inergativos -véanse (12) y (13), respectivamente-:

(11) estable, mejorable, variable vs. *gritable, *nadable, *sonreible

(12) bebedor, corredor, trabajador vs. *aparecedor, *estallador, *moridor

(13) durmiente, participante, sonriente vs. *estallante, *enfermante, *enrojeciente

\subsection{VEA y VCE: definición y contraste}

Comenzamos definiendo el grupo de los VEA sirviéndonos de la acertada explicación de Mendikoetxea (1999: 1607): "se pueden describir como 'cobrar existencia'». De esta afirmación se deduce la diferencia esencial entre los VEA y los VCE. Los primeros, (14), seleccionan un argumento sujeto con función semántica de Tema, que no existe previamente al evento ${ }^{3}$, siendo similar al Objeto Efectuado de los verbos transitivos de creación ( $\mathrm{Su}$ padre construyó la casa; Marcos ha hecho un pastel riquísimo; La empresa ha fabricado un nuevo dispositivo). Los segundos, (15), asignan la función sintáctica de sujeto (de Tema o Tópico para algunos lingüistas) a argumentos cuya entidad ya existía antes del desarrollo del evento, pues expresan el cambio de estado que dicha entidad experimenta, característica que se aprecia especialmente bien en aquellos verbos con variante anticausativa:

(14) Su hijo nació de madrugada / Han aparecido las llaves / Aroa viene pronto

(15) Se ha parado el reloj / Has crecido mucho / La maceta cayó desde muy alto

Ambos tipos de verbos presentan otra diferencia bastante arraigada: la que contempla que los VEA exigen un argumento locativo, mientras que los VCE solo añaden locaciones adjuntas: El pájaro llegó a la ventana /_Samanta viene a la oficina vs. La planta ha florecido (a la orilla del río) / Samanta palideció (en la oficina). Creemos que incluso es posible concebir que los VEA generan en primer lugar el actante espacial, a partir del cual se va a desarrollar el evento, esto es, la existencia o aparición del sujeto: El pájaro llegó a la ventana $\rightarrow$ la ventana [Locación] + LLEGAR el pájaro [Sujeto]; Samanta viene a la oficina $\rightarrow$ la oficina [Locación] + VENIR Samanta [Sujeto].

En cuanto a los VCE, se trata de un grupo que, igual que ocurriera con los VEA, cuenta con dos subclases que se pueden concentrar bajo una única denominación: la formada por los llamados verbos de cambio de estado puros y la formada por los llamados verbos de

3 Es importante relacionar las nociones de existencia y aparición: la primera atiende al hecho de que, al menos en un mundo posible, hay una entidad como la que denota el verbo; la segunda, a su surgimiento empíricamente percibido por el hablante. Así, existir o vivir se consideran verbos de existencia y llegar o nacer, verbos de aparición. No obstante, ambas clases comparten suficientes propiedades léxico-sintácticas para propiciar su denominación conjunta. 
cambio de ubicación. Demonte (1994) define ambos grupos a partir del objeto nocional que seleccionan. Así, los verbos de cambio de estado puros designan un Objeto que cambia de estado «cuando la materia que lo conforma experimenta alguna modificación» (Demonte, 1994: 73), mientras que los Objetos de los verbos de cambio de ubicación no varían su composición material, sino el lugar físico o mental que ocupan (v. Demonte, 1994: 48); añade el matiz de que los de cambio de ubicación son verbos que seleccionan un Objeto que varía su locación mediante la acción de un Agente. Puntualicemos esta idea: consideramos que se trata de una explicación muy útil para los verbos transitivos con Agente y Tema, verbos a partir de los cuales se forman los inacusativos de variante anticausativa -la mayor parte de los VCE-, pero creemos que esto no solo afecta a los de cambio de ubicación, sino a todo el conjunto de los VCE, otro motivo por el que conviene reunir sus dos subclases: en El leñador taló el árbol no hallamos un cambio de ubicación, sino un cambio de estado, e igualmente la acción es ejecutada por un Agente y ejercida sobre un Tema, como sucede en El leñador movió la leña. Creemos que para el estudio de la inacusatividad no es relevante ramificar la etiqueta de Tema en otras como Paciente-Objeto Afectado (el árbol), Objeto Desplazado (la leña), etc., por lo tanto, el comportamiento léxico-sintáctico de ambas subclases es idéntico, no resultando oportuno sostener que haya dos etiquetas distintas para los VCE, por mucho que, extralingüísticamente, sea apreciable que no es lo mismo modificar materialmente un objeto que cambiarlo de lugar.

Como hemos comentado, una de las propiedades características de los VCE es que, salvo excepciones, su configuración sintáctica permite secuencias anticausativas con el clítico se con valor medio, propiedad de la que carecen los VEA, puesto que no se originan a partir de verbos bivalentes:

(16) La criada secó la ropa / La ropa se secó

(17) * La criada nació la niña / * La niña se nació

Los VCE con variante anticausativa presentan una inacusatividad derivada, relacionada con la pasivización de las oraciones activas transitivas. Según Buzelin Haro (2009: 6), los verbos de cambio de ubicación no aceptan esta variante anticausativa, solo lo hacen los de cambio de estado material. En nuestra opinión, esto es un contraargumento falso con la finalidad de discernir entre ambas subclases de VCE. Si un verbo no presenta variante anticausativa es porque no existe su correspondiente pronominal que pueda aportarla: existe secar y existe secarse, pero también existen mover y moverse (El leñador movió la leña $\rightarrow$ La leña se movió), que denotan cambio de ubicación; existe crecer, pero no crecerse entendido literalmente, como existe partir y no existe partirse, entendido como VM. Es una cuestión que compete al léxico de cada verbo, no una regla general de la caracterización de los verbos de cambio de estado material frente a los de cambio de ubicación.

\section{El problema de los VM}

Consideramos que en español solo existen cuatro predicados que deban ser clasificados como VM: entrar, ir, llegar y salir, creyendo irrelevante la distinción entre Verbos de Dirección Inherente (VD) y Verbos de Manera de Moverse (VMM). 
Creemos que los VMM son VD que seleccionan una configuración concreta en torno a la noción de manera, es decir, los VMM se reducen a VD que seleccionan el rasgo [+ manera] una vez considerada la opción [ \pm manera] en su Estructura de Qualia (EQ a partir de ahora) (v. Pustejovsky, 1995 y De Miguel, 2009). Lapesa y Lenci (2012) ofrecen un interesante corpus de VMM italianos que muestran esa especificación; tomándolo como modelo, citamos algunos ejemplos en español, representativos de los diferentes hiperónimos:

(18) Variedades de andar: anadear, bailar, zigzaguear

Variedades de correr: esprintar, galopar, trotar

Movimiento sigiloso: deslizarse

Movimiento rápido fuera del centro deíctico: escaparse, huir, volar

Movimiento rápido hacia una meta: asaltar, embestir, irrumpir

Movimiento de progreso gradual: arrastrarse, gatear, reptar

¿Sería factible descomponer, por ejemplo, andar como ir + rasgos específicos de andar, escaparse como salir + rasgos específicos de escaparse, embestir como llegar + rasgos específicos de embestir o irrumpir como entrar + rasgos específicos de irrumpir? A nuestro juicio, sí; de hecho, hemos llegado a la conclusión de que los VM se deducen todos a partir de cuatro primitivos semántico-cognitivos: entrar en, ir hacia, llegar hasta y salir de. Una prueba de ello es que los VD y los VMM que se crean a raíz de estos verbos solo pueden construirse con SSPP introducidos por las mismas preposiciones que rigen ellos:

(19) entrar en: aterrizar en, embarcarse en, irrumpir en, penetrar en...

ir hacia: avanzar hacia, bajar/subir hacia, caminar hacia, dirigirse hacia...

llegar hasta: acudir hasta, extenderse hasta, regresar hasta, venir hasta...

salir de: distanciarse de, huir de, irse de, volar de...

No obstante, para que este análisis pueda ser correcto, conviene tener en cuenta que es necesario que el VM seleccione el rasgo [+ trayectoria] en su EQ. Por otro lado, respecto a los VD que denotan curso o fondo del movimiento, creemos que se reducen a llegar hasta + rasgo [+ manera] + rasgo [+ trayectoria], siendo el rasgo [+ trayectoria] el final del Objeto-Lugar. Asimismo, si bien los VM que consideramos primitivos a nivel semánticocognitivo rigen una determinada preposición y, en consecuencia, se construyen con un tipo de argumento de trayectoria concreto (Ubicación, Trayectoria, Meta u Origen, respectivamente), pueden seleccionar otras preposiciones (con sus correspondientes argumentos) en usos recategorizados. Así, entrar puede ser también entrar hasta e ir puede ser también ir hasta; sus derivados, salvo excepciones ${ }^{4}$, presentan la misma posibilidad: irrumpir hasta, penetrar hasta / correr hasta, dirigirse hasta, por ejemplo. Cifuentes-Férez (2008: 393-431) ofrece un corpus con doscientos cincuenta y siete VM en español Menciona los

4 No es posible construir aterrizar hasta o embarcarse hasta, por ejemplo, pues aterrizar y embarcarse solo permiten la interpretación canónica de entrar en, relacionada con una superación de límite no prolongable: entrar en (Ubicación) $\rightarrow$ entrar hasta (Ubicación como Tema Incremental; dada una Ubicación, Ubicación en punto $1 \rightarrow$ Ubicación en punto 2) vs. [aterrizar / embarcarse] en (Ubicación) $\rightarrow *$ [aterrizar / embarcarse] hasta (Ubicación indivisible). De igual forma, Fernández Leborans considera que construcciones del tipo entrar dentro o entrar ahí implican previamente entrar en. 
rasgos que componen la red léxica de cada uno. Hemos recopilado a continuación cuántos verbos se relacionan con cada uno de los posibles componentes semánticos ${ }^{5}$ :

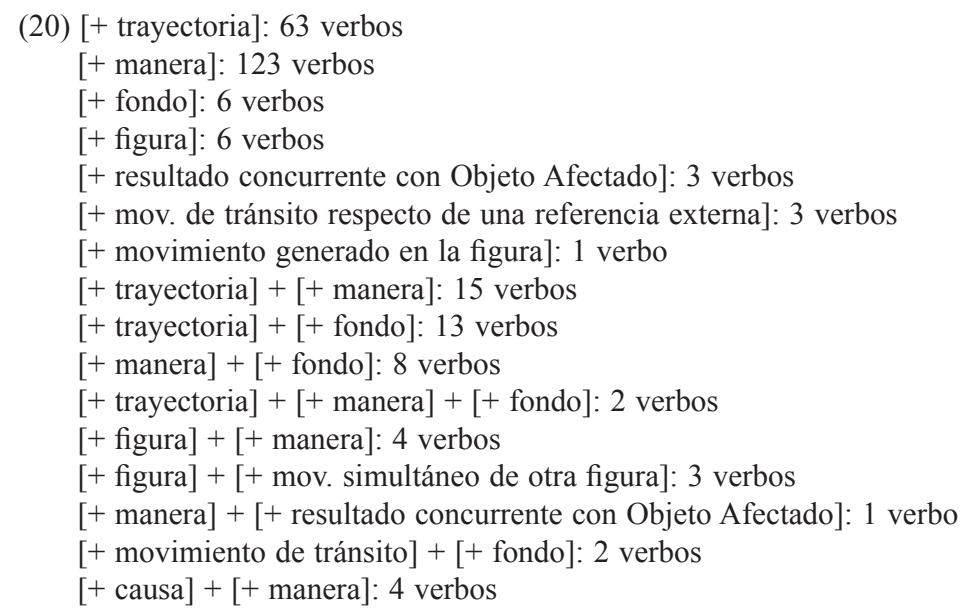

El 24,5\% corresponde a verbos que únicamente seleccionan el rasgo [+ trayectoria], mientras que el $47,8 \%$ corresponde a verbos que solo seleccionan el rasgo [+ manera]. Estos datos muestran que en español hay un buen número de VM que se interpretan como VD y que casi el doble son considerados VMM. Creemos que los VD españoles están consolidando en su clase, y que a partir de ellos se generan los VMM. Continuando con esta idea, como anticipamos antes, creemos que hay cuatro VD primitivos semántico-cognitivos, a partir de los cuales se constituye el resto. Como vimos mediante el modelo de descomposición de Lapesa y Lenci (2012), esto mismo ocurre con los VMM, que especializan su rasgo [+ manera], atendiendo a lo que Ibarretxe-Antuñano y Caballero (2014: 146), siguiendo a Slobin (1996), llaman VMM de primer orden y VMM de segundo orden, respectivamente, siendo, pues, simplemente, VD derivados que focalizan dicho rasgo. Ilustramos nuestra exposición con la siguiente relación entre predicados:

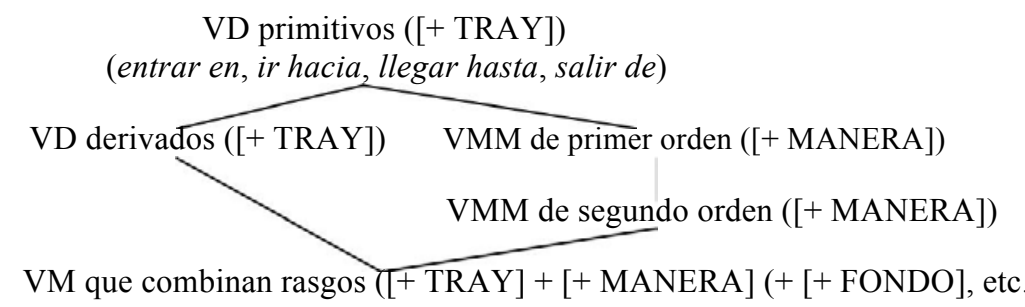

Teniendo presente lo comentado hasta ahora sobre los VM, parece viable considerar que se trata de un grupo bastante concreto dentro de nuestra gramática. Sin embargo, y partiendo de la propuesta que acabamos de defender, ¿no resulta innecesario mantener una

5 Para un estudio de la tipología utilizada en dicha clasificación, véase Talmy (1985). 
etiqueta unánime para un grupo de predicados que apenas cuenta con cuatro representantes? Asimismo, ¿dichos representantes muestran las mismas características en cuanto al Modo de Acción o la inacusatividad - por citar algunos factores de evaluación común- o solo se establece su uniformidad terminológica a través de la laxa noción de movimiento? No nos ocuparemos en estas páginas de este segundo problema; centrándonos en el primero, creemos que establecer una categoría (autónoma, se sobreentiende), dentro del marco de una lengua debe estar justificado ya desde el número de elementos que la conforman, porque, entre otras cosas, de no ser así, dicha categoría se convierte en una especie de cajón de sastre adonde van a parar elementos que no han llegado a ser descritos con eficiencia durante los diferentes estudios que se les haya podido dedicar. Con todo ello, consideramos que los VM son VCE (concretamente, verbos de cambio de ubicación): Entrar, ir, llegar y salir no expresan sino un cambio de lugar de la entidad, que abandona una ubicación para ocupar otra: Entidad en $\mathrm{X}+\mathrm{V} \rightarrow$ Entidad en $\mathrm{Y}$.

\section{El verbo Llegar}

Es de reseñar que al principio de este trabajo pusimos llegar como ejemplo de predicado perteneciente al grupo de los VEA (El pájaro llegó a la ventana), considerando que el sujeto "hace aparición" en un determinado lugar en un determinado momento, interpretando, entonces, -basándonos en la ausencia de volitividad al no poseer el rasgo [+ humano]que no lleva a cabo dicha aparición de manera intencional. Esta secuencia es semejante, pues, a El paquete llegó al buzón o a El anticiclón llegó al interior de la península, por ejemplo. Lo interesante ahora es saber si construcciones como Victoria llegó hasta Madrid, en las que el sujeto sí puede presentar el rasgo [+ volitivo], se corresponden con predicados del tipo VEA o del tipo VCE. A nuestro juicio, hay pruebas que demuestran que en español hay dos acepciones del verbo llegar, esto es, la interpretación inacusativa y la interpretación inergativa de llegar se corresponden con sentidos independientes. Así, construcciones como Victoria llegó hasta casa y como El paquete llegó al buzón atienden a interpretaciones diferentes debido a la coacción que ejercen los diferentes factores que operan en uno y otro predicado.

En Zagona (2006: 174-175) ya se plantea esta idea: la agramaticalidad de secuencias como Llegaron profesores y lo hicieron estudiantes también «puede ser debida a la incongruencia entre el tipo de argumento seleccionado por llegar (Tema) y el tipo de argumento seleccionado por hacerlo (Agente) (...) Una posibilidad es que verbos como llegar tengan dos entradas léxicas: una entrada inacusativa y otra entrada agentiva, que selecciona un Agente como argumento externo normal»; entonces Los profesores llegaron y los estudiantes también lo hicieron es plausible porque «el papel de Agente puede ser solamente un papel externo, asignado composicionalmente por SV. Ambos miembros de la coordinación tienen un verbo agentivo», mientras que Llegaron profesores y lo hicieron estudiantes también no lo es porque «hacerlo se analizaría como agentivo, pero el argumento interno de llegaron en el primer miembro de la coordinación no puede ser Agente, puesto que es un argumento interno, como lo pone de manifiesto la falta de determinante». A continuación ilustramos esta propuesta con las pruebas a las que aludíamos antes y que, en nuestra opinión, dan cuenta, no de dos piezas léxicas distintas, pero sí de dos acepciones distintas de llegar: 
(22)

\begin{tabular}{|c|c|}
\hline$\underline{\text { Llegar }}^{1}$ & $\underline{\text { Llegar }} \underline{\underline{2}}$ \\
\hline Inergativo & Inacusativo \\
\hline VCE de Ubicación & VEA \\
\hline Sujeto Agente & Sujeto Tema \\
\hline Predicado de Realización & Predicado de Logro \\
\hline Trayectoria con hasta / a & Locación con $a$ \\
\hline Imperativo (llégate $)$ & NO Imperativo $(* l l e g a)$ \\
\hline Adjuntos de carácter volitivo & NO Adjuntos de carácter \\
\hline \multicolumn{2}{|l|}{ Forma llegador } \\
\hline Victoria llegó hasta casa & Victoria llegó a casa \\
\hline *El verano llegó hasta Madrid & El verano llegó a Madrid \\
\hline \multicolumn{2}{|l|}{ \#El paquete llegó hasta la } \\
\hline oficina & El paquete llegó a la oficina \\
\hline \#El huracán llegó hasta la isla & El huracán llegó a la isla \\
\hline
\end{tabular}

El llegar inergativo expresa un predicado de cambio de ubicación con un sujeto Agente, que lleva a cabo el desplazamiento desde una ubicación X hasta una ubicación Y, desplazamiento que atiende a un proceso [+ durativo] y [+ télico], esto es, a un evento de Realización, cuya trayectoria es introducida por la preposición hasta o, de manera alternativa, por la preposición $a$, sin que entre ellas se produzca un cambio de significado en este contexto ${ }^{6}$. Frente al llegar inergativo, el llegar inacusativo expresa un predicado de existencia o aparición con un sujeto Tema, que experimenta la acción, no controlando el evento, que carece de duratividad y se corresponde con un predicado de Logro, caracterizado exclusivamente como [+ télico], siendo la preposición $a$ la que introduce el locativo en el que se produce la "aparición" del Tema.

Conviene que profundicemos en varias cuestiones: en primer lugar, creemos que llegar es una excepción a los VCE del tipo de ubicación, dado que el cambio de ubicación se produce de manera voluntaria por parte del sujeto, circunstancia que nos lleva a pensar que incluso pueda ser conflictivo considerar que llegar es un VCE, en el sentido de que es posible que los VM no cumplan con las propiedades que se presuponen para este grupo de verbos, pues entrar, ir o salir tampoco seleccionan de forma natural sujetos que no sean Agentes. En este mismo grupo de VCE se encuentran verbos como caer, que sí expresa un cambio de ubicación no controlado por su argumento sujeto. Lo que queremos decir es que tal vez no sea oportuno proponer una sub-clasificación de los VCE si en realidad esta presenta notables excepciones, como hemos demostrado que ocurre con los VM. No obstante, a falta de un estudio más exhaustivo de esta clase de verbos, en este trabajo hemos optado por utilizar esta categoría para los predicados de movimiento, pues nos resulta inadecuado concluir que los VM forman un grupo autónomo dentro de la Gramática. Sea de un modo o de otro, más allá de dificultades terminológicas, lo relevante para nuestra propuesta es que este tipo de verbos se opone a los VEA en varias de sus características,

6 Según Fernández Leborans (c. p.), la frecuencia de uso de la preposición $a$ se debe a su subespecificación -propiedad también apreciable en la preposición de-; desde el latín posee significado neutro direccional, en el sentido de orientación a un límite final. Al tratarse de un significado por defecto, puede adoptar valores como el de hasta. 
presentadas en estas páginas, de entre las cuales ahora es especialmente pertinente recordar aquellas que aluden a los rasgos que poseen sus sujetos y sus argumentos locativos.

Así, el sujeto de llegar ${ }^{1}$ y la trayectoria introducida por hasta conforman un predicado de Realización en el que el Agente cumple con la Actividad que denota el proceso durativo previo al Logro expresado por el límite espacial, esto es, la ubicación de destino, el punto último de la trayectoria. Expuesto de manera sencilla, esta interpretación de llegar se ilustraría como sigue:

(23) [Ubicación ${ }_{1}\left[\right.$ Agente ir ([+ durativo]) hasta ([+ télico]) [llegar [Ubicación $\left.\left.\left.\left.{ }_{2}\right]\right]\right]\right]$

Por el contrario, llegar ${ }^{2}$, inacusativo, presenta su condición de VEA a través de la manifestación del sujeto Tema en el locativo introducido por la preposición $a$. Dicho locativo representa una Locación, no una Trayectoria, pues no se produce un cambio de ubicación, sino una "aparición" en un lugar dado, por lo que se produce una acción relativamente puntual o instantánea, correspondiente a un predicado de Logro 7 . Con todo ello, el evento expresado por llegar $^{2}$ podría ilustrarse así:

(24) [Locación [(aparición de ([+ télico])) [Tema]]]

Ambas representaciones manifiestan el contraste entre el sujeto con rasgo [ + humano] y [+ volitivo] (Agente, llegar $^{1}$ ) y el sujeto con rasgo [- animado] y [- volitivo] (Tema, llegar ${ }^{2}$. Sin embargo, existen usos de uno y otro verbo que requieren de una explicación en este contexto comparativo, como son los casos siguientes:

\section{(25) a. Victoria llegó a casa \\ b. El paquete llegó hasta la oficina \\ c. El huracán llegó hasta la isla}

La interpretación natural de (25a) es agentiva, con la preposición $a$ cumpliendo con el valor direccional de hasta. No obstante, es posible entender que Victoria "hace aparición" en el lugar expresado por el locativo. Si considerásemos esta opción, claramente marcada, el sujeto cancelaría su rasgo [+ volitivo] ${ }^{8}$ y se convertiría en un Tema semántico desde el punto de vista temático; por tanto, Victoria tendría la misma condición que el verano, el paquete o el huracán en los contextos de VEA. En (25b) y en (25c), llegar hasta es comparable a alcanzar ${ }^{9}$, en tanto en cuanto el sujeto recorre una trayectoria limitada por el locativo expreso. No obstante, conviene matizar la comparación entre ambas secuencias: en (25b) entendemos que el paquete llega a un lugar que no era el esperado, en tanto que la preposición añade un sentido de desplazamiento mayor que supera el destino previsto (destino que se marcaría con $a$ en el caso natural de esta secuencia, como VEA). Sin embargo, en (25c)

7 En Pérez Jiménez y Moreno Quibén (2007) se trata con detalle la estricta relación existente entre el fenómeno de la Inacusatividad y la noción aspectual de Telicidad.

8 Este tipo de contextos demuestra que el rasgo [+ volitivo] es relevante para definir la agentividad, pues ni el rasgo de animacidad ni específicamente el rasgo [+ humano] son suficientes para describir semánticamente como Agente a un argumento.

9 A este respecto, observa García Fernández (c. p.) que en estos contextos la preposición hasta presenta un matiz de alcance o consecución sobre el verbo. 
la preposición no aporta esa connotación, el locativo representa igualmente el punto final del desplazamiento, pero no podemos decir que se trate de un destino no previsto, porque, de hecho, en este contexto no hay un destino previsto, sino un punto de culminación en el que concluye la acción después de haber pasado por otros puntos durante el desplazamiento (el huracán se crea en X, pasa por Y, llega hasta Z, donde desaparece, tratándose entonces de un Tema Incremental). Regresando a sus construcciones canónicas, si representamos sintácticamente la comparación entre $l$ legar ${ }^{1}$ y llegar ${ }^{2}$, obtendremos el siguiente contraste ${ }^{10}$ :

(26) a. Victoria llegó hasta Madrid:

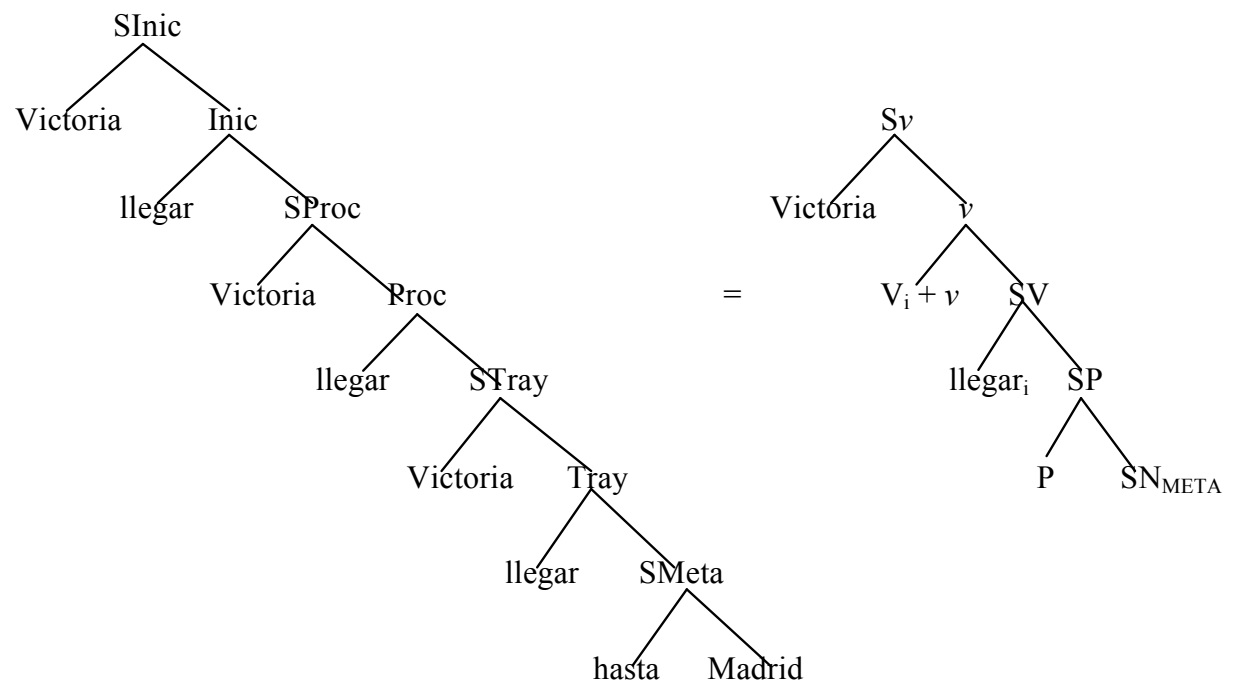

b. El verano llegó a Madrid:
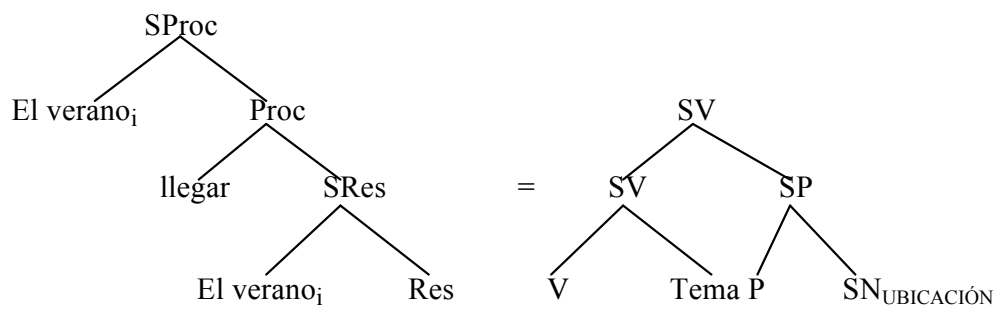

En segundo lugar, las diferencias entre ambos predicados (llegar ${ }^{1}$ inergativo y llegar ${ }^{2}$ inacusativo) se manifiestan también a través de varios aspectos morfológicos y sintácticos. Creemos que se ha venido considerando que llegar se correspondía con una única interpretación, y que esta era inacusativa, por lo que su forma imperativa no sería posible, como no es posible de manera natural con ningún verbo que no seleccione como sujeto un Agente:

10 Nos basamos en los modelos representacionales de Ramchand (2008) y Jiménez-Fernández y Tubino (2013) y de Kratzer (1996), respectivamente. 


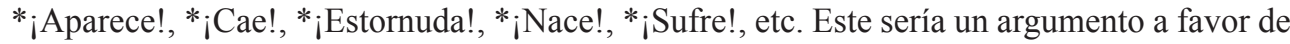
considerar que llegar $^{2}$ no tiene, pues, forma de imperativo. Sin embargo, llegar ${ }^{1}$ sí presenta dicha forma, pues se comporta como inergativo, siendo su sujeto capaz de controlar la acción expresada por el predicado, como sucede con los predicados inergativos y transitivos: ¡Come!, ¡Corre!, ¡Entra!, ¡Mira!, ;Sal!, ;Trabaja!, etc. La peculiaridad que presenta llegar $^{1}$ frente a aquellos otros es que requiere del clítico no argumental. Así, *iLlega! sería la forma imperativa de llegar ${ }^{2}$, no aceptable, mientras que ;Llégate! correspondería a llegar ${ }^{1}$. En nuestra opinión, esta forma sería semejante a la imperativa de ir hasta o de acercarse: Llégate hasta la tienda $\rightarrow$ Ve hasta la tienda /Acércate a la tienda, donde el clítico aporta la agentividad del evento -ligada a la Actividad que expresa- y la preposición, su telicidad, formando el predicado de Realización que denota llegar ${ }^{1}$.

En cuanto a los complementos adjuntos de carácter volitivo, parece clara la oposición entre llegar ${ }^{1}$ y llegar $^{2}$ respecto a su inclusión; solo el primero, al ser agentivo, los acepta: Victoria llegó hasta casa a propósito vs. *El verano llegó a Madrid para molestarnos; *El paquete llegó a la oficina deliberadamente; *El huracán llegó a la isla aposta.

Finalmente, queda por explicar la posibilidad de la formación del derivado agentivo llegador a partir de llegar ${ }^{1}$. Hemos investigado la aceptación y el uso de este término, considerando su existencia como argumento a favor de nuestra propuesta de la distinción entre dos acepciones distintas para llegar. A continuación exponemos nuestras conclusiones al respecto.

Para empezar, llegador formaría parte del primer grupo sugerido por Cano (2010: 5) en alusión a los derivados de verbos inergativos e inacusativos: «tenemos verbos cuyo adjetivo derivado en -dor selecciona lecturas dinámicas y el derivado por medio de -nte selecciona una lectura estativa». A primera vista, como observamos en el apartado dedicado al diagnóstico de Inacusatividad, parece una doble prueba bastante atractiva considerando que los adjetivos acabados en -ble pueden modificar a sujetos cuyo papel semántico es Tema, propio de los inacusativos, y los sustantivos construidos con los sufijos -nte y -dor son solo válidos orientados a un sujeto Agente, el que seleccionan los inergativos. Sin embargo, fracasaremos si intentamos extender tales juicios a todos los verbos inacusativos e inergativos ${ }^{11}$ :

(27a) *nacible, *aparecible, *entrable vs. saltable, tragable, respirable

(27b) Formaciones inacusativas: ardiente, creciente; naciente...

(27c) hervidor, llegador, bajador ${ }^{12}$ vs. *llorador, *bostezador, *cruzador

Así las cosas, las previsiones de esta prueba no se cumplen dadas las numerosas excepciones que presenta, siendo llegador una de ellas. Pérez Jiménez (2006: 282) explica su restricción así: «ni los verbos de 'dirección inherente' y 'cambio de estado' que denotan Logros desde el punto de vista del Modo de Acción, ni tampoco los verbos de 'aparición, desaparición o suceso' dan lugar a derivados en -dor / -tor. Se trata por tanto de verbos inacusativos». Campos (1999: 1567) utiliza también dicho diagnóstico para confirmar la

11 Instamos al lector a acercarse al trabajo de Val Álvaro (1981) sobre el sufijo -ble y al trabajo de Laca (1993) sobre los sufijos -dor y -nte.

12 «Llegador y bajador son términos comunes en el ciclismo» (Cifuentes Honrubia, 1999: 49, nota 11). 
agramaticalidad de formas como *idor, *venidor, *moridor, *partidor o *salidor. En la NGLE (2009: § 6. 7c) se dice lo siguiente: «En ocasiones, estas voces provienen de los verbos llamados inacusativos en contextos en los que denotan ciertas acciones de carácter intencional, lo que hace pensar que han dejado de pertenecer propiamente a esta clase sintáctica», y se citan los ejemplos de llegador, entrador y salidor.

Aportamos ahora las siguientes opiniones acerca de esta aceptación de llegador $^{13}$ : Bosque (c. p.): «Tal como dice la NGLE, es agentivo en esos contextos, puesto que llegar se convierte aquí en una actividad, hasta el punto de que se refiere a cierto tipo de acción especializada en algunos deportes»;

Pérez Jiménez (c. p.):

«Efectivamente, llegador existe para el ciclismo, y lo único sensato que se puede decir es que hay un llegar agentivo: Juan llegó tarde porque le dio la gana; Juan llegó a la meta el último deliberadamente -distinto, pues, de llegar en El tren llega o El agua llega hasta la cintura $^{14}$ - y a partir de ahí se forma llegador, que se ha especializado»;

Campos (c. p.):

«Es posible entender llegador como entrador (chico que tiene éxito con las chicas). En todo caso no hay un verbo para eso, por lo que no creo que se pueda hablar de derivación como en trabajador. En entrador o llegador se requiere de una habilidad que va más allá del sentido (¿figurado?) del verbo. Me gusta la explicación de que hay una recategorización del predicado en Actividad, que puede hacer que sea más Agente. Porque puedes decir "ese chico es entrador con las chicas ricas a propósito", indicando que hay agentividad de por medio».

Yendo un paso más allá en la descripción de llegador como término especializado, investigamos sobre su uso en el ámbito del fútbol. Descubrimos que no se trata de un calco del inglés, pues en inglés se utiliza la lexía 'box to box' ${ }^{15}$, sino que se ha acuñado directamente en nuestra lengua: «Es un llegador con instinto, con mucho desmarque, que sabe buscarse la vida en los balones largos y al que nunca se le achica la portería (Mundo [Esp.] 9/2005)» (tomado de la NGLE, 2009: § 6. 7c). Así pues, un llegador llega, igual que un escritor escribe o un corredor corre, con ello queremos decir que no se trata de un uso de carácter metafórico o figurado; atiende a una determinada acción llevada a cabo de manera voluntaria por parte de un Agente, por lo que no es posible aplicarlo a entidades con rasgo [- animado], de modo que en esos contextos solo es plausible la interpretación inergativa de llegar, esto es, el llegar agentivo cuyo sujeto controla el evento.

13 Agradecemos a Ignacio Bosque, Isabel Pérez Jiménez y Héctor Campos su tiempo y sus observaciones.

14 En este contexto, en el que también tendrían cabida ejemplos como La capa llega hasta la rodilla o La raja llega hasta el techo, llegar se mostraría próximo a una interpretación de Estado (v. De Miguel y Fernández Lagunilla, 2007).

15 «En inglés su equivalente es 'box to box', que es el centrocampista que domina todo el campo y llega con peligro a posiciones de ataque». Agradecemos esta observación a Proyecto Premier, expertos en fútbol inglés y su terminología. 


\section{Conclusiones}

Hemos considerado oportuno estudiar con mayor exhaustividad el caso del verbo llegar, dadas sus asimetrías respecto de los demás predicados analizados en este trabajo (VCEs, VEAs y VMs). Hemos tratado su comportamiento atendiendo a ciertos patrones, mostrando su carácter variable en contra de su apriorística concepción como inacusativo $\mathrm{y}$, mediante factores como la derivación nominal o el clítico se, entre otros, hemos podido ilustrar paralelamente nuestra propuesta de que la Inacusatividad en español debe ser considerado un fenómeno heterogéneo en cuanto a la relación léxico-sintaxis.

Hemos concluido que la particularidad del verbo llegar radica en que hay pruebas de índole semántico-sintáctica que demuestran que se trata de una pieza léxica con, al menos, dos acepciones distintas, correspondientes a dos interpretaciones codificadas léxico-sintácticamente: una en la que se comporta como un VEA y otra en la que se comporta como un VCE (de Ubicación), próximo a los VM por defecto - grupo dentro del que consideramos irrelevante la distinción VD-VMM, creyendo que existen cuatro verbos (entrar en, ir hacia, llegar hasta y salir de) de carácter primitivo en sentido semántico-cognitivo, a partir de los cuales se constituyen tanto VD como VMM según sus matices-.

El primer sentido de llegar semejante a aparecer, que selecciona un locativo encabezado por la preposición $a$ y cuyo sujeto es Tema semántico. Se trata de un predicado de Logro, inacusativo, no admite complementos que expresen volición ni permite la forma de imperativo, tampoco la construcción con el clítico se. El segundo, por el contrario, expresa un cambio de ubicación entre dos locaciones; dicho cambio de ubicación se debe al desplazamiento del sujeto Agente, que controla la acción. Se construye con la preposición hasta, que da cuenta del carácter incremental del evento, confirmando, por tanto, su definición como predicado de Realización. Es inergativo, permite la inclusión de adjuntos que aporten volición y la construcción con el clítico agentivo, así como la forma de imperativo. A partir de él se construye llegador, término que refleja una propiedad derivada de una Actividad, correspondiente a una determinada acción dentro del ámbito de ciertos deportes. Paralelamente, hemos demostrado que, si bien su uso puede estar restringido a ciertos contextos, es un término acuñado en español, no tomado de la adaptación de uno equivalente en otro idioma, hecho que refuerza nuestra propuesta de que en nuestra lengua existen dos acepciones de llegar que, por lo tanto, deben ser descritas de manera independiente, demostrando, pues, que el fenómeno de la Inacusatividad debe ser interpretado desde un prisma que se ajuste a una mayor flexibilidad caracterizadora de los predicados.

\section{Bibliografía}

Batiukova, O. (2004): "Sobre la intransitividad y la estructura subeventiva de los verbos de movimiento", en Interlingüística 15.1, 177-188.

Bosque, I. y Gutiérrez-Rexach, J. (2009): Fundamentos de sintaxis formal, Madrid, Akal, 6. 4. y 6. 9. Burzio, L. (1981): Intransitive verbs and italian auxiliaries, Cambridge, MIT Press.

Burzio, L. (1986): Italian Syntax: A Government-binding Approach, Dordrecht, Reidel.

Buzelin Haro, C. M. (2009): Los verbos de cambio. Una aproximación léxico-semántica y aspectual, Universidad Nacional de Córdoba, Facultad de Filosofía y Humanidades, Escuela de Letras.

Campos, H. (1999): “Transitividad e intransitividad”, en I. Bosque y V. Demonte (eds.): Gramática descriptiva de la lengua española, Madrid, Espasa-Calpe, cap. 25, 1519-1574. 
Cano, M. Á. (2010): "La interpretación de causa y agente en las formas deverbales en -nte”, en 39 Simposio de la Sociedad Española de Lingüística, Universidad de Santiago de Compostela.

Chomsky, N. (2006): “Approaching UG from below”, manuscrito, MIT, Mass.

Chomsky, N. (2013): "Problems of Projection", en Lingua 130, 33-49.

Cifuentes-Férez, P. (2008): Motion in English: a perspective from Cognitive Linguistics, Typology and Psycholinguistics, Tesis Doctoral, Universidad de Murcia.

Cifuentes Honrubia, J. L. (1999): “Inacusatividad y movimiento", en Revista Española de Lingüística $29,35-61$.

Deal, A. M. (2009): “The origin and content of expletives: Evidence from selection", Syntax, 12(4): 285-323, University of Harvard.

De Miguel, E. (2009): "La Teoría del Lexicón Generativo", en E. de Miguel (ed.): Panorama de la Lexicología, Barcelona, Ariel, cap. 5, 337-368.

De Miguel, E. y Fernández Lagunilla (2007): "Sobre la naturaleza léxica del aspecto composicional", en P. Cano López (coord.): Actas del VI Congreso de Lingüistica General, Santiago de Compostela 3-7 de mayo de 2004, Vol. 2, Tomo 1: Las lenguas y su estructura (IIa), 1767-1778

Demonte, V. (1994): "La semántica de los verbos de cambio", en B. Garza Cuarón, J. A. Pascual y A. Alonso González (coords.): II Encuentro de lingüistas de España y México (1991), 535-563.

Fernández Leborans, M. J. (2005): Los sintagmas del español II. El sintagma verbal y otros, Madrid, Arco-Libros.

Ibarretxe-Antuñano, I. y Caballero, R. (2014): "Una aproximación al estudio de los eventos de movimiento metafórico desde la tipología semántica y el género", en Anuari de Filologia. Estudis de Lingüística, 4, 139-155.

Jiménez-Fernández, A. L. y Tubino, M. (2013): “Causativización de intransitivos y variación lingüística", en Taller de Variación Sintáctica del Español: SEL, CSIC.

Kratzer, A. (1996): "Severing the external argument from its verb", en J. Rooryck y L. Zaring (eds.): Phrase structure and the lexicon, Dordrech, Kluwert Publications, 109-137.

Laca, B. (1993): "Las nominalizaciones orientadas y los derivados españoles en -dor y -nte", en S. Varela (comp.), La formación de palabras, Taurus.

Lapesa, G. y Lenci, A. (2012): "Italian Verbs of Manner of Motion at the Syntax-Semantics Interface: a Distributional Analysis", Linguistic Evidence, Tubingen.

Mendikoetxea, A. (1999): “Construcciones inacusativas y pasivas" en I. Bosque y V. Demonte (eds.): Gramática descriptiva de la lengua española, Madrid, Espasa-Calpe, cap. 26, 1575-1630.

Pérez Jiménez, I. (2006): La gramática de las cláusulas absolutas de predicación en español, Tesis Doctoral, UCM, 4. 3.

Pérez Jiménez, I. y Moreno Quibén, N. (2007): “¿Son todos los verbos inacusativos aspectualmente télicos en español? El papel de la telicidad en la interficie léxico-sintaxis", en Cano et al. (eds.): Actas del VI Congreso de Lingüística General, Madrid, Arco-Libros, 1807-1820.

Perlmutter, D. M. (1978): "Impersonal passives and the unacussativity hypothesis", Proceedings of the $4^{\text {th }}$ annual meeting of the Berkeley Linguistic Society, 157-189.

Pustejovsky, J. (1995): The Generative Lexicon, Cambridge, MIT Press.

Ramchand, G. (2008): Verb Meaning and the Lexicon: A First-Phase Syntax, Cambridge, Cambridge Studies in Linguistics 116.

Real Academia Española y Asociación de Academias de la Lengua Española (2009): Nueva Gramática de la Lengua Española, Madrid, Espasa Libros, vol. II: Morfología. Sintaxis I.

Slobin, D. I. (1996): "Two ways to travel: Verbs of motion in English and Spanish", en M. Shibatani y S. A Thompson (eds.): Grammatical Constructions: Their Form and Meaning, Oxford. Clarendon Press, 195-219.

Talmy, L. (1985): "Lexicalization patterns: Semantic structure in Lexical Forms", en T. Shopen (ed.): Language Typology and Syntactic Description, vol. 3: Grammatical Categories and the Lexicon, 57-150. 
Torrego, E. (1989): “Unergative-unaccusative alternations in Spanish”, MIT Working Papers in Linguistics, 10, 253-272.

Val Álvaro, J. F. (1981): "Los derivados sufijales en -ble en español”, en Revista de Filología Española, vol. $61, \mathrm{~N}^{\circ} 1 / 4$.

Villar, F. (1983): Ergatividad, acusatividad y género en la familia lingüística indoeuropea, Ediciones Universidad de Salamanca y F. Villar, Salamanca.

Zagona, K. (2006): Sintaxis generativa del español, Madrid, Visor Libros, traducción al castellano de The syntax of Spanish (2002), Cambridge, Cambridge University Press. 